\title{
Reproducing human arm motion using a kinematically coupled humanoid shoulder-elbow complex
}

\author{
M.M. Stanišić and C.M. Goehler* \\ Department of Aerospace and Mechanical Engineering, University of Notre Dame, Notre Dame, IN 46556, USA
}

(Received 14 August 2008; final version received 29 September 2008)

\begin{abstract}
A challenge that is gaining interest in robotics is the development of humanoids, which are robots that assume an anthropomorphic form. A difficulty with humanoid design is the kinematic interpretation of human joints and the development of mechanisms that can mimic human motion. The focus of this work is the development of a kinematic description of the shoulder-elbow complex. A mechanism capable of reproducing voluntary human reaching motions is introduced along with the procedural method of implementing the coupled motions that exist within the human shoulder complex and shoulderelbow complex. Experimental results are presented highlighting the accuracy of this mechanism along with the similarities to human configurations.
\end{abstract}

Keywords: humanoid robotics; inverse kinematics; shoulder-elbow coupling; method of successive iterations

\section{Introduction}

It is believed that the first articulated anthropomorphic robot in the history of western civilization was created between 1495 and 1497 by Leonardo da Vinci (Rosheim 1997). It looked like a suit of armour and was cable driven. Currently, the inspiration to design humanoid robots is the same as Leonardo's: to use anatomical, kinesiological and mechanical studies to create a machine identical in function to a human being.

There have been several humanoid shoulder complexes designed in the recent years. Most of these were developed as subsystems of larger systems. One such humanoid robotic shoulder design can be seen in NASA's Robonaut (Ambrose et al. 2000; Nickels and Baker 2003). The Robonaut utilizes a 7-DOF (degrees of freedom) arm with the shoulder represented as three revolute joints connected in a serial chain. This design takes into account the contributions of the spherical glenohumeral joint, excluding the effects of the shoulder girdle.

A similar shoulder design can be found in the Dexter robot arm (Zollo et al. 2002). The Dexter arm consists of an anthropomorphic 8-DOF kinematic structure with the shoulder represented by the first three revolute joints, again only accounting for the contributions of the spherical glenohumeral joint. The unique aspect of the Dexter arm is the drive system. The arm is not actuated by motors on each link, but instead is driven by a system of pulleys and steel cables with the motors installed on the first link. This allows the Dexter arm to generate human-like movements but results in a heavy, dynamically complex primary joint.

Asfour et al. (2000) proposed the design for the humanoid robot ARMAR with the desire to achieve a high degree of mobility and to accommodate direct cooperation with humans. The ARMAR robot also utilises a 3-DOF serial chain as the shoulder complex, in this case accounting for some motion within the shoulder girdle along with the glenohumeral joint.

The HRP-2 is a humanoid robotics platform with the ability of whole body motion (Kaneko et al. 2002, 2004). Whereas the Robonaut existed from the torso up and the ARMAR robot maneuvered via a wheel-driven platform, the HRP-2 utilises biped locomotion. This model was designed with the intent of fully mimicking total human motion. The HRP-2 uses three revolute joints to represent the shoulder complex similar to the previously mentioned designs, also only accounting for the glenohumeral joint.

Nishiwaki et al. (2000) presented the design and development of the humanoid robot H6. Similar to the HRP-2, this robot was designed to be compact and light weight. The H6 accounts only for the glenohumeral joint and models the shoulder as 3-DOF.

The Humanoid Robotics Institute at Waseda University developed two humanoid robots with the goal of cohabitation with humans (Hashimoto et al. 2002). Hadaly-2 was designed to realise information and physical interactions with humans while remaining gentle and safe. WABIAN has a complete human configuration with the ability to

*Corresponding author. Email:c-goehler@northwestern.edu 
walk on two legs. Both models utilise a 7-DOF arm where the shoulder is modelled as 3-DOF.

The Sony QRIO is a small biped entertainment robot (Ishida 2004; Sawada 2004; Tanaka and Suzuki 2004; Movellan et al. 2005; Tanaka et al. 2005). The humanoid utilises touch sensing and motion sensing systems for safety and entertainment. The goal is for QRIO to be a partner robot and to behave spontaneously and emotionally. This adds to its human-like behaviour. QRIO also uses a 3-DOF joint to model the shoulder.

More recently, groups have begun to design mechanisms that more accurately mimic human motion in both function and appearance. Okada et al. $(1999,2001)$ proposed a new 3-DOF parallel mechanism, named the Cybernetic Shoulder, that integrates human-like high mobility and human-like sensitive compliance into the design. This mechanism combines the effective motion of the shoulder girdle and the glenohumeral joint into one 3-DOF mechanism. This cybernetic shoulder mechanism was modified and implemented into the design of the CREST humanoid (Nakamura et al. 2002; Okada et al. 2003). The goal of this humanoid was to design mechanisms that maximise whole body mobility while being limited to the space of a human-shaped body. Using the cybernetic shoulder mechanism, CREST was able to produce a similar range of motion to that of the human shoulder.

Rosheim (1997) designed the Robotic Surrogate with an added 2-DOF in the shoulder (making it a 5-DOF mechanism) to account for scapula-clavicle motion. This design was inspired by Leonardo's Vitruvian Man drawings, which clearly showed that a spherical joint alone could not reproduce the motion of the arm without cheating. A similar design exists in the WE-4R robot (Miwa et al. 2004). Miwa et al. developed a humanoid robot arm with the desire for the WE-4R robot to show emotions in a better way. They added two rotational DOF to the shoulder joint, similar to the Surrogate, in order to allow it to square and shrug its shoulders. In both designs, these additional DOF help to reposition the shoulder centre of the typical 7-DOF robot arm.

Sodeyama et al. (2005) proposed a humanoid design that imitates the structure of the human shoulder, especially the scapula. This model, named Blade, uses a musculoskeletal torso with the scapula driven by motor-actuated tendons. This design is the most similar to a human but is difficult to power, especially with increasing loads on the arm. It can also be difficult to find effective muscle layouts and then the model cannot achieve the desired shoulder motions.

One design that was pursued primarily to replicate the human shoulder complex used a combination of a parallel mechanism which serves as the shoulder girdle and a serial mechanism which serves as the spherical glenohumeral joint (Lenarcic et al. 2000; Stanisic et al. 2001; Lenarcic and Stanisic 2003). These two subsystems operate as an offset double pointing system. This design is one of the few shoulder mechanisms that models the shoulder as more than just a ball-in-socket joint. Klopcar and Lenarcic (2001) presented a study comparing the ranges of motion of the human shoulder complex and the previously discussed parallel mechanism using the Elite Motion Analyser. The recorded data displayed that the humanoid robotic shoulder was able to fulfill a similar range of motion of the human shoulder girdle during movement executed in the three cardinal planes.

There have been many advances in the design of total humanoid systems as seen in the aforementioned models, but little has been done with robot shoulders. There are very few differing humanoid shoulder designs. Aside from a few models, most humanoid robotic shoulder designs utilise the typical 3-DOF model and do not take into account all aspects of motion in the human shoulder complex, namely the motion of the scapula, and none of the models account for the coupling between the shoulder complex and the orientation of the elbow joint. This study builds upon the shoulder complex composed of a combination of a parallel mechanism and serial mechanism seen in Lenarcic et al. (2000), Stanisic et al. (2001) and Lenarcic and Stanisic (2003) and extends it into a whole arm design.

\section{Definition of coordinate system and anatomical planes}

Three-dimensional analysis of human body kinematics requires a coordinate system, typically composed of anatomically aligned axes as shown in Figure 1: medial/lateral (ML), anterior/posterior (AP) and superior/inferior (SI) (Oatis 2004). The following Cartesian coordinate system is used throughout this paper: the $X$-axis is aligned with the ML axis with positive $X$ in the medial direction, the $Y$-axis is aligned with the SI axis with positive $Y$ in the superior direction and the $Z$-axis is aligned with the $\mathrm{AP}$ axis with positive $Z$ in the anterior direction. This coordinate system will be referred to as the anatomical body frame.

In the human body, there are three perpendicular anatomical planes referred to as the cardinal planes. The sagittal plane is the $Y-Z$ plane, the frontal plane is the $X-Y$ plane and the transverse plane is the $X-Z$ plane. These planes are also displayed in Figure 1 (Oatis 2004).

\section{The human shoulder complex}

The shoulder complex is comprised of three moving bones: the clavicle, the scapula and the humerus, and one fixed structure: the sternum/thorax (rib cage). There are four joints: sternoclavicular, acromioclavicular, scapulothoracic and glenohumeral as seen in Figure 2 (Lenarcic et al. 2000; Oatis 2004). The sternoclavicular joint can be classified as a ball-in-socket joint connecting the sternum and the clavicle. The acromioclavicular joint acts as a ball-in-socket 


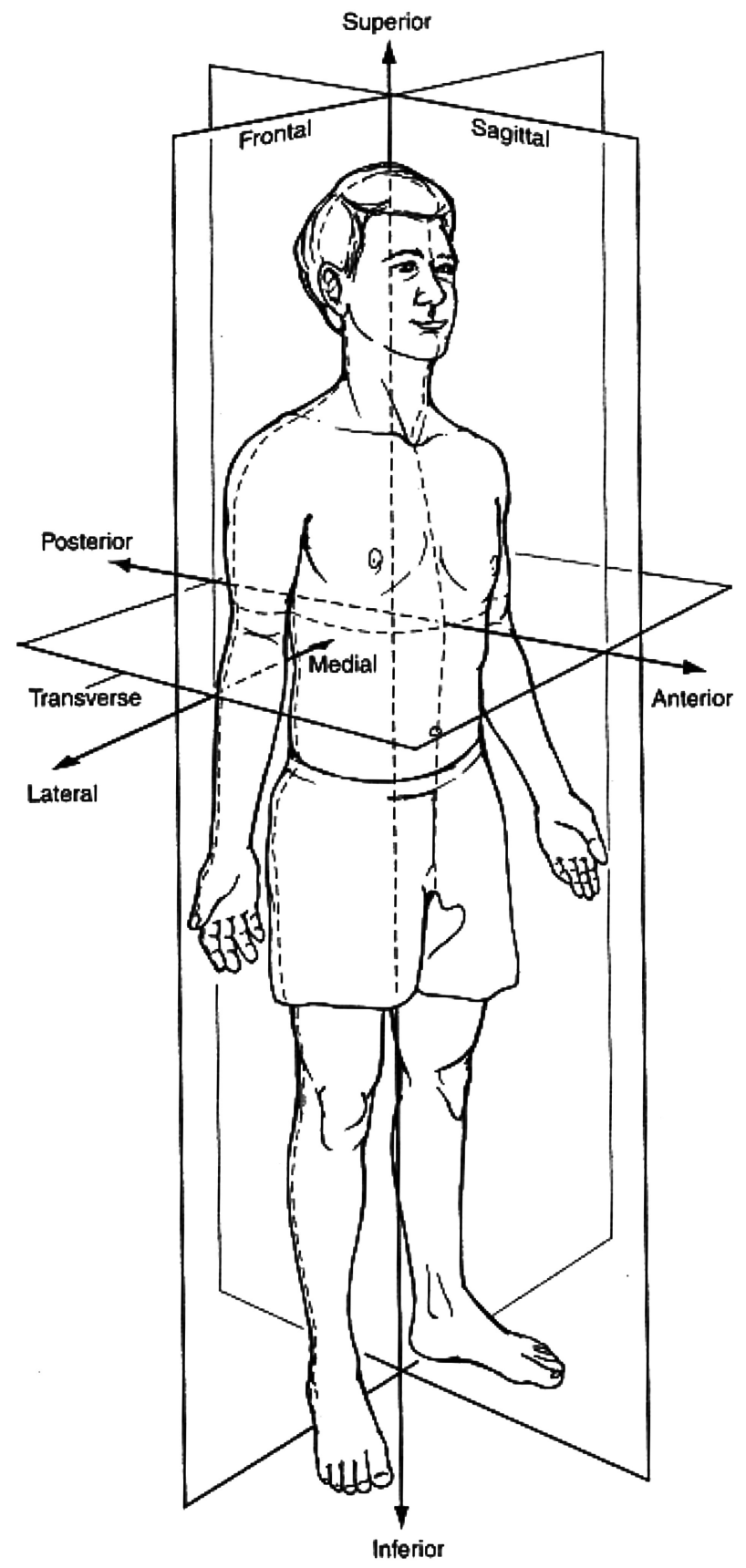

Figure 1. Definition of cardinal planes (Oatis 2004).

joint between the clavicle and the scapula. The scapula and the thorax are not directly attached through the scapulothoracic joint, but rather are separated by muscles allowing for systematic and repeated motion. The overall motion of the scapulothoracic joint includes two translations and two rotations used primarily to amplify the motion of the glenohumeral joint. One of the rotations is a pitch off of the thorax seen only in unnatural movements such as shrugging and is therefore disregarded when kinematically modelling the scapulothoracic joint. The glenohumeral joint is the most

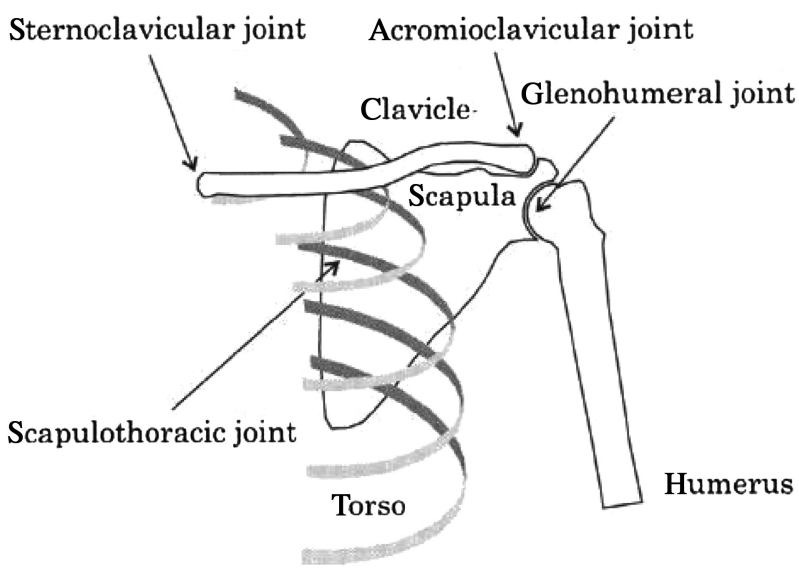

Figure 2. Shoulder complex (Lenarcic et al. 2000).

mobile ball-in-socket joint in the human body. Its amplified mobility challenges the inherent stability of the joint which is provided by non-bony structures, primarily the rotator cuff which is a cartilaginous muscle.

\subsection{Degrees of freedom in the shoulder complex}

It is important to determine the number of degrees of freedom in a system in order to understand how it moves. The shoulder complex has three moving bodies, each contributing 6-DOF of motion to the complex, giving the shoulder complex 18-DOF of possible motion. For each of the three ball-in-socket joints (sternoclavicular, acromioclavicular and glenohumeral seen in Figure 3), 3-DOF are removed from the complex due to the constraints of the joints. This brings the total possible degrees of freedom down to 9-DOF. The scapulothoracic joint can be modelled either as a planar joint (as indicated in Figure 3) or as another ball-in-socket joint with the centre of curvature near infinity. In both cases, this joint also removes 3-DOF from the total due to the constraints of the joint. The total degrees of freedom of the shoulder complex is reduced to 6-DOF. Of these 6-DOF, one is the trivial spinning of the clavicle which can be neglected. A second degree of freedom is the humeral spin which is similar to a robotic end

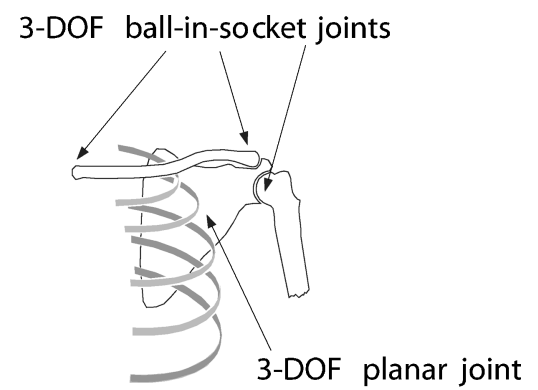

Figure 3. Degrees of freedom of the shoulder complex. 
effector roll and does not affect the direction of the humerus. That leaves 4 DOF to determine the direction of the humerus.

\subsection{The two subsystems of the shoulder complex}

Examining only the shoulder girdle comprised of two moving bones (clavicle and scapula) and one fixed structure (sternum/thorax) shows a closed kinematic chain with 12DOF of available motion. The three joints of the girdle (sternoclavicular, acromioclavicular and scapulothoracic) each remove 3-DOF resulting in a 3-DOF closed chain system. Neglecting the trivial spin leaves 2-DOF used for pointing the central axis of the glenoid fossa (the socket on the scapula that the humeral head lies in to produce the glenohumeral joint).

Thus, the shoulder girdle uses 2-DOF to point the central axis of the glenoid fossa of the glenohumeral joint and the glenohumeral joint uses the other 2-DOF to point the humerus. These two systems are displaced due to the geometry of the bones and joints. Kinematically, the shoulder complex is therefore comprised of two pointing systems separated by an offset and a twist (Lenarcic and Stanisic 2003). This is an important point which is the basis for the humanoid shoulder-elbow complex mechanism developed in this paper.

\section{Humanoid shoulder-elbow mechanism}

Figure 4 shows the humanoid shoulder-elbow complex mechanism considered here. It is an extension of the shoulder complex of Lenarcic and Stanisic (2003) into a whole arm which includes an elbow. It is called a shoulder-elbow complex because as in a human, the direction of the elbow axis is coupled to the motion of the shoulder when voluntary (i.e., natural or unforced) reaching motions are performed. It consists of two subsystems: the shoulder girdle mechanism and the serial arm mechanism. The shoulder girdle consists of two orthogonally intersecting revolute joints. The serial arm mechanism consists of the glenohumeral joint, whose centre is at the intersection of three successively orthogonal revolute joints, and the elbow joint, which is a single revolute joint. The kinematic equations for this mechanism are developed using the Denavit and Hartenberg (DH) parameters given in Table 1. The frame assignments are seen in Figure 4, following the procedure discussed in Craig (1989). The $\hat{Y}_{i}$ axes follow the $\hat{X}_{i}$ and $\hat{Z}_{i}$ axes and are not shown. Frames $\{T\}$ and $\{W\}$ represent the base frame located at the thorax and the end effector frame located at the wrist, respectively. Frame $\{S\}$ is introduced as the shoulder frame located at the shoulder centre. This frame is used to relate the two subsystems. $\hat{X}_{S}$ represents the central axis of the glenoid fossa.

DH parameters $a_{0}$ and $a_{2}$ represent the combined lengths of the shoulder girdle bones used to determine
Table 1. Denavit and Hartenberg parameters for the shoulderelbow complex mechanism.

\begin{tabular}{ccccc}
\hline$i$ & $\alpha_{i-1}$ & $a_{i-1}$ & $d_{i}$ & $\theta_{i}$ \\
\hline 1 & $\frac{\pi}{2}$ & $a_{0}$ & 0 & $\theta_{1}$ \\
2 & $\frac{\pi}{2}$ & 0 & 0 & $\theta_{2}$ \\
$S$ & $\alpha_{2}$ & $a_{2}$ & 0 & 0 \\
3 & 0 & 0 & 0 & $\theta_{3}$ \\
4 & $\frac{\pi}{2}$ & 0 & 0 & $\theta_{4}$ \\
5 & $\frac{\pi}{2}$ & 0 & $d_{5}$ & $\theta_{5}$ \\
6 & $\alpha_{5}$ & 0 & 0 & $\theta_{6}$ \\
7 & 0 & $a_{6}$ & 0 & 0 \\
\hline
\end{tabular}

the distance from the sternum to the glenohumeral joint centre. DH parameter $\alpha_{2}$ represents the twist that exists between the two pointing systems of the shoulder complex (initially shown as $\alpha_{2}=180^{\circ}$ in the figure). DH parameters $d_{5}, a_{6}$ and $\alpha_{5}$ represent the length of the humerus, the length of the forearm and the angular offset of the elbow axis, respectively. $\alpha_{5}$ has a value between $-100^{\circ}$ and $-105^{\circ}$ to accommodate the human elbow's 'carrying angle' (Zatsiorsky 1998).

\section{Inverse kinematics}

The inverse kinematics problem of the humanoid shoulderelbow complex mechanism is defined as: given the coordinates of the wrist in the base frame, $\bar{P}$ in Figure 4,

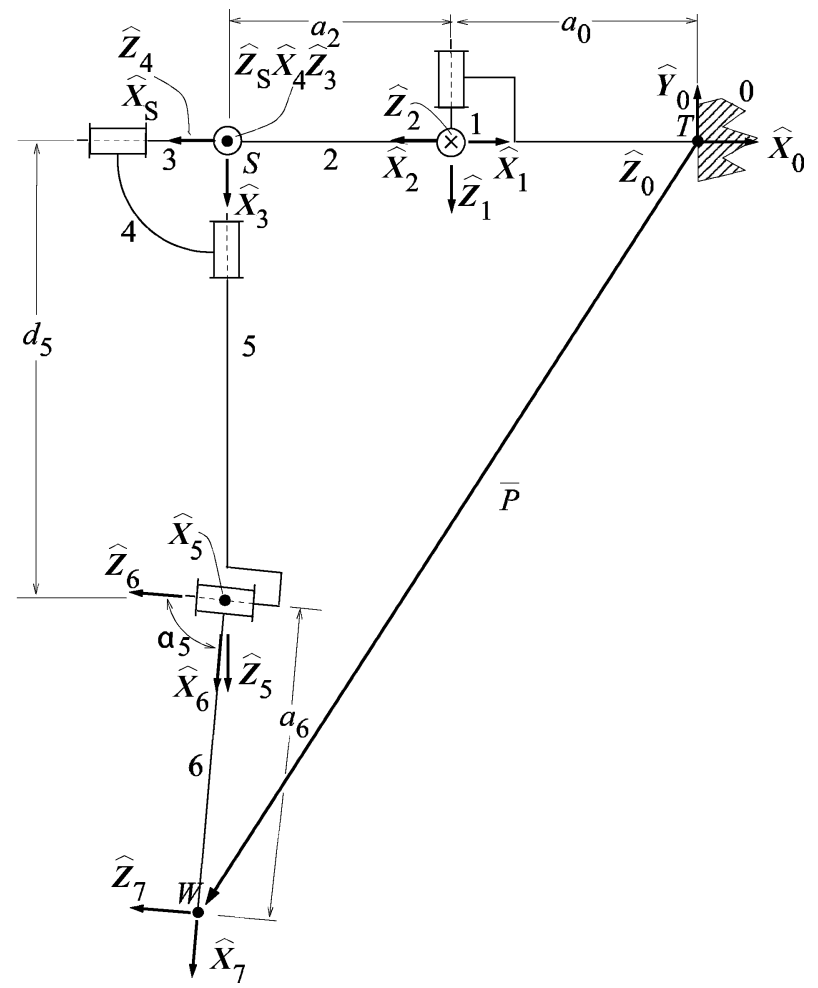

Figure 4. Humanoid shoulder-elbow complex mechanism. 
determine the values of the joint variables $\theta_{1}-\theta_{6}$, a problem with three equations in six unknowns, i.e., three redundant degrees of freedom. Three constraints need to be imposed to resolve the redundancy and more importantly, these constraints must conform to human shoulder-elbow complex motions.

Two constraints which couple the first four degrees of freedom come from Lenarcic and Stanisic (2003). These constraints approximate the 2:1 ratio between human humeral and scapular rotation. Another two constraints come from Stanisic et al. (2007), which couple the direction of the elbow axis to the reaching direction, for voluntary human-reaching motions. Reaching direction is defined as the unit vector directed from the centre of the glenohumeral joint to the wrist centre. Implementing these two pairs of couplings will allow the humanoid shoulder-elbow complex to replicate voluntary human-reaching motion. However, there are now four constraints imposed, leading to an over-constrained system. Furthermore, with the couplings, the system of equations is too complex for a closed-form inverse kinematics solution.

In the inverse kinematics the two pairs of couplings can be separated from each other, since the effect of one pair of couplings on the other pair is weak. The weak nature of this coupling allows the inverse kinematics problem to be solved using a method of successive iterations. Dobrowolski (1965) gives an example of a complex system of equations broken into two independent subsystems of weakly coupled equations, solved by successively iterating between the two systems until the complete solution converges. This method allows the inverse kinematics problem to be solved as follows, given the coordinates of the wrist in the base frame $\bar{P}$.

Step 1: Set $\theta_{1}=0^{\circ}$ and $\theta_{2}=180^{\circ}$. This corresponds to a solution which neglects scapula motion.

Step 2: Solve the inverse kinematics problem for $\theta_{3}-\theta_{6}$, implementing the coupling between reaching direction and elbow axis direction found in voluntary human reaching described by Stanisic et al. (2007).

Step 3: Knowing $\theta_{1}-\theta_{4}$, determine the direction of the humerus $\hat{Z}_{5}$.

Step 4: Calculate new values for $\theta_{1}$ and $\theta_{2}$ using $\hat{Z}_{5}$, implementing the coupling between human humerus and scapula rotation found in Lenarcic and Stanisic (2003).

Step 5: Iterate Steps 2-4 until the change in the values of the joint angles satisfies a convergence criterion. Iterations are required because the solution in Step 4 will affect the location and orientation of the glenohumeral joint which will alter the reaching direction in Step 2.

The following section adds some details to these steps.

\subsection{Successive iteration solution to the inverse kinematics problem}

For the starting point of the desired trajectory, the shoulder girdle angles are initially set to,

$$
\theta_{1}=0^{\circ}, \quad \theta_{2}=180^{\circ},
$$

which corresponds to an inverse kinematics solution that neglects scapular motion. For successive points along a wrist trajectory, the final shoulder girdle angles of the previous point will be used. The value $\alpha_{2}=120^{\circ}$ is used in this inverse kinematics solution as was used in Lenarcic and Stanisic (2003) (equivalent to their constant $\theta_{3}$ variable). This completes Step 1.

Let $\bar{P}=\left[P_{x}, P_{y}, P_{z}\right]^{T}$ represent the desired coordinates of the wrist centre in frame $\{0\}$ and ${ }^{S} \bar{P}=\left[{ }^{S} P_{x},{ }^{S} P_{y},{ }^{S} P_{z}\right]^{T}$ represent the coordinates of the wrist center in frame $\{S\} .{ }^{S} \bar{P}$ is calculated from $\bar{P}$ in Equation(1),

$$
{ }^{S} \bar{P}={ }_{S}^{0} T^{-1} \bar{P},
$$

where ${ }_{S}^{0} T$ is the homogeneous transformation known from the values of $\theta_{1}$ and $\theta_{2}$.

The equations for ${ }^{S} \bar{P}$ come from concatenating the homogeneous transformations from frame $\{7\}$ to frame $\{S\}$. The vector ${ }^{S} \bar{P}$ is the position vector in the fourth column of ${ }_{7}^{S} T$ (shorthand, $s_{i}=\sin \theta_{i}, c_{i}=\cos \theta_{i}, s \alpha_{i}=\sin \alpha_{i}$ and $\left.c \alpha_{i}=\cos \alpha_{i}\right)$

$$
\left[\begin{array}{c}
{ }^{s} P_{x} \\
{ }^{s} P_{y} \\
{ }^{s} P_{z}
\end{array}\right]=\left[\begin{array}{c}
a_{6} s_{3} A+c_{3}\left(s_{4} B+a_{6} c_{4} C\right) \\
-a_{6} c_{3} A+s_{3}\left(s_{4} B+a_{6} c_{4} C\right) \\
-c_{4} B+a_{6} s_{4} C
\end{array}\right]
$$

where

$$
\begin{aligned}
& A=s_{5} c_{6}+c \alpha_{5} c_{5} s_{6}, \\
& B=d_{5}+a_{6} s \alpha_{5} s_{6}, \\
& C=c_{5} c_{6}-c \alpha_{5} s_{5} s_{6} .
\end{aligned}
$$

The values of $\theta_{3}-\theta_{6}$ are calculated from a known ${ }^{S} \bar{P}$ in Equation (2), implementing the two constraints from Stanisic et al. (2007) which relate the elbow axis direction to the reaching direction, or the glenohumeral-elbow axis coupling (GE coupling for short). This results in an overconstrained system of five equations in four unknowns, isolating the over-constrained portion of the inverse kinematics problem.

Before dealing with the over-constrained problem, it is possible to solve for the elbow angle $\theta_{6}$, which depends only on the magnitude of ${ }^{S} \bar{P}$. Summing the squares of the $x, y$ 
and $z$ components of Equation (2) and solving for $\theta_{6}$ yields

$$
\theta_{6}=\arcsin \left(\frac{{ }^{s} P_{x}{ }^{2}+{ }^{s} P_{y}{ }^{2}+{ }^{s} P_{z}{ }^{2}-a_{6}{ }^{2}-d_{5}{ }^{2}}{2 a_{6} d_{5} s \alpha_{5}}\right) .
$$

Because of the limitations in human elbow articulation, only one solution for $\theta_{6}$ from Equation (3) is retained.

The solution for $\theta_{3}-\theta_{5}$ is where the voluntary-reaching problem is over-constrained by the GE coupling. The goal here is to find the solution to this over-constrained problem. Two of the three angles can be found using the equations (2), while the third will be optimised to determine the best approximation of the GE coupling. The procedure will be as follows.

First, the reaching direction, $\hat{u}=\left[u_{x}, u_{y}, u_{z}\right]^{T}$, is calculated as the normalised ${ }^{S} \bar{P}$ vector rotated into the anatomical body frame

$$
\hat{u}={ }_{S}^{0} R \frac{{ }^{S} \bar{P}}{\left|{ }^{S} \bar{P}\right|},
$$

where ${ }_{S}^{0} R$ is the rotation matrix in ${ }_{S}^{0} T$, and ${ }_{S}^{0} T$ is known from $\theta_{1}$ and $\theta_{2}$. In order to use the GE coupling as given by Stanisic et al. (2007), $\hat{u}$ is converted to two angles (spherical coordinates) defining the reaching direction $\left(\phi_{\text {reach }}, \theta_{\text {reach }}\right)$,

$$
\begin{gathered}
\phi_{\text {reach }}=\operatorname{atan} 2\left(u_{y}, \sqrt{u_{x}^{2}+u_{z}^{2}}\right), \\
\theta_{\text {reach }}=\operatorname{atan} 2\left(\frac{u_{x}}{\cos \phi_{\text {reach }}}, \frac{u_{z}}{\cos \phi_{\text {reach }}}\right) .
\end{gathered}
$$

Then from Stanisic et al. (2007), two angles defining the elbow axis direction $\left(\phi_{\text {elbow }}, \theta_{\text {elbow }}\right)$, can be determined. This elbow axis direction is then converted back to a unit vector in the anatomical body frame

$$
\hat{V}_{\text {desired }}=\left[\begin{array}{c}
V_{x_{\text {desired }}} \\
V_{y_{\text {desired }}} \\
V_{\text {zdesired }}
\end{array}\right]=\left[\begin{array}{c}
\cos \phi_{\text {elbow }} \sin \theta_{\text {elbow }} \\
\sin \phi_{\text {elbow }} \\
\cos \phi_{\text {elbow }} \cos \theta_{\text {elbow }}
\end{array}\right] .
$$

Equation (6) gives the direction of the humanoid's elbow axis direction which would replicate voluntary humanreaching motions according to Stanisic et al. (2007).

To get a minimum norm error solution to this overconstrained problem, the value of $\theta_{5}$ is fixed and then $\theta_{3}$ and $\theta_{4}$ are computed from Equation (2). Using ${ }^{S} P_{z}, \theta_{4}$ can be solved using the tangent of the half angle formulas, resulting in two values of $\theta_{4}$. Using ${ }^{S} P_{x}$ and ${ }^{S} P_{y}$, values for $\mathrm{s}_{3}$ and $\mathrm{c}_{3}$ can be calculated, resulting in one value of $\theta_{3}$ for each set of $\theta_{4}-\theta_{6}$. This results in two solution sets, however, only one solution set will result in an outward elbow direction (similar to a human) and is therefore the only feasible solution. For this set of $\theta_{3}-\theta_{5}$ values, the direction of the humanoid's elbow axis, ${ }^{0} \hat{Z}_{6}$, is computed from the forward kinematic equations. The norm of the error between this elbow axis direction and that from Stanisic et al. (2007) in Equation (6) is then determined.

The human arm is capable of achieving humeral rolls of approximately $180^{\circ} . \theta_{5}$ is incremented through this range and the set of $\theta_{3}-\theta_{5}$ that minimises the norm of the error in the elbow axis direction is found. This completes Step 2.

The direction of the humerus, ${ }^{0} \hat{Z}_{5}=\left[x_{5}, y_{5}, z_{5}\right]^{T}$, is determined from the forward kinematics using Equation (7),

$$
\begin{aligned}
& {\left[\begin{array}{l}
x_{5} \\
y_{5} \\
z_{5}
\end{array}\right]} \\
& =\left[\begin{array}{c}
s_{1}\left(s \alpha_{2} c_{3} s_{4}-c \alpha_{2} c_{4}\right)-c_{1}\left[c_{2} s_{3} s_{4}+s_{2}\left(c \alpha_{2} c_{3} s_{4}+s \alpha_{2} c_{4}\right)\right] \\
s_{2} s_{3} s_{4}-c_{2}\left(s \alpha_{2} c_{4}+c \alpha_{2} c_{3} s_{4}\right) \\
-c_{1}\left(s \alpha_{2} c_{3} s_{4}-c \alpha_{2} c_{4}\right)-s_{1}\left[c_{2} s_{3} s_{4}+s_{2}\left(c \alpha_{2} c_{3} s_{4}+s \alpha_{2} c_{4}\right)\right]
\end{array}\right],
\end{aligned}
$$

which completes Step 3.

The inverse kinematic solution for the shoulder complex uses the procedure outlined by Lenarcic and Stanisic (2003) to compute the joint angles $\theta_{1}$ and $\theta_{2}$ from ${ }^{0} \hat{Z}_{5}$, completing Step 4.

It is now possible to recalculate ${ }^{S} \bar{P}$ using Equation (1) where ${ }_{S}^{0} T$ uses these new values of $\theta_{1}$ and $\theta_{2}$. Steps 2-4 are repeated until the solutions for $\theta_{1}$ through $\theta_{6}$ have converged. This completes Step 5.

The final values of $\theta_{1}$ and $\theta_{2}$ and the dimension $\alpha_{2}$ can be used to determine the values of the leg lengths $l_{1}, l_{2}$ and $l_{3}$ in the parallel shoulder girdle mechanism which actuate these angles, again following the procedure discussed in Lenarcic and Stanisic (2003).

\section{Evaluation of humanoid shoulder-elbow complex motion}

With the kinematics of the humanoid shoulder-elbow complex mechanism established, it is necessary to evaluate the resulting motion in comparison to actual human arm motion. This can be done both quantitatively and qualitatively by comparing the configuration of the mechanism to that of the human arm.

\subsection{Quantitative comparison of humanoid mechanism motion to human motion}

The data from the human motion studies in Stanisic et al. (2007) was utilised to attain the quantitative comparison. The mechanism dimensions were scaled to match the subject for each comparison. The mean difference between the reaching direction of the mechanism and the actual reaching direction from the data was $12.08^{\circ}$ with a standard deviation of $8.64^{\circ}$. The mean difference between the elbow axis direction of the mechanism and the actual elbow axis direction from the data was $20.96^{\circ}$ with a standard deviation of $11.56^{\circ}$. 
At first glance, these numbers may not seem ideal. Considering the fact that there exists some error in the marker positions (outlined in Stanisic et al. 2007) and the fact that there are three optimisation problems being utilised to solve the inverse kinematics, the results are not as bad as they may first appear. The first optimisation problem occurs in using the surface fits from the regression analysis to calculate the elbow axis direction from the reaching direction (Stanisic et al. 2007). These surfaces are best fits of a finite set of data and therefore can only give the best, or optimal, elbow axis direction for a given reaching direction. The second optimisation problem occurs in solving for the three angular rotations at the shoulder, $\theta_{3}-\theta_{5}$. This process selects the arm configuration that has the smallest difference between the desired elbow axis direction acquired from the surface fits and the actual elbow axis direction calculated during the inverse kinematics solution. The errors that arise from doing each of these optimisation problems are now accumulating.

The third optimisation problem occurs while determining the inverse kinematics of the shoulder girdle that results in the humeral direction that changes the least amount. This process has the greatest impact on the numerical results presented earlier. One of the largest contributors to error during this particular process is the fact that $a_{0}$ and $\alpha_{2}$ are taken to be constants in the mechanism. In reality the distance between the glenohumeral joint and the sternum changes during arm motions. This leads to the computed shoulder centre being in a different location than the actual shoulder centre obtained from the data. This, in turn, results in the computed reaching direction of the mechanism being different than that from the data. The choice of $\alpha_{2}$ also impacts the solutions for $\theta_{3}-\theta_{5}$ which in turn affects the computed reaching direction. Ideally, the value of $\alpha_{2}$ would be variable and would be optimised during each arm motion in order to best fulfill the operating criteria presented in Lenarcic and Stanisic (2003). This error then propagates throughout since a different reaching direction is being used to calculate the elbow axis direction using the surface fits and then the arm kinematics are further optimised around a very different desired elbow axis direction. This can be

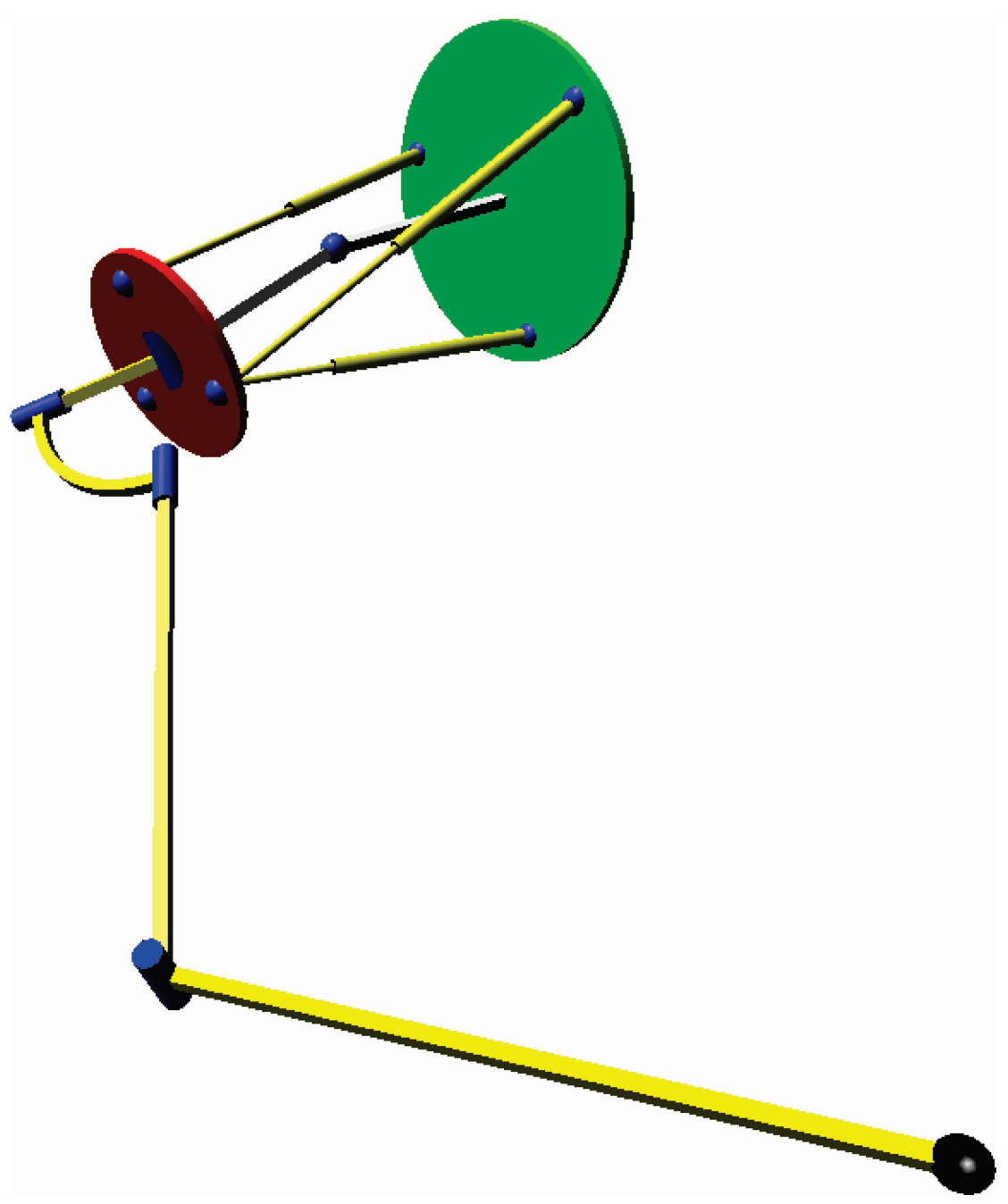

Figure 5. The humanoid mechanism animation. 
(a)
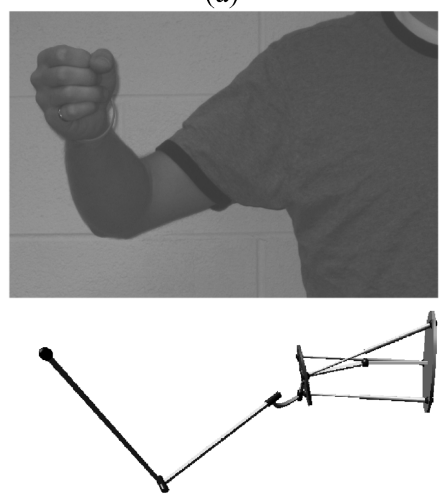

(c)
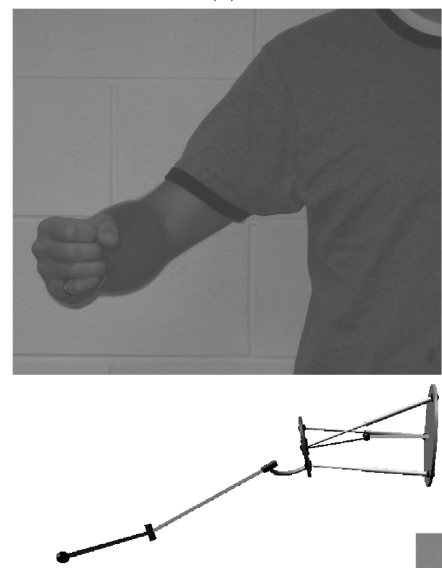

(b)
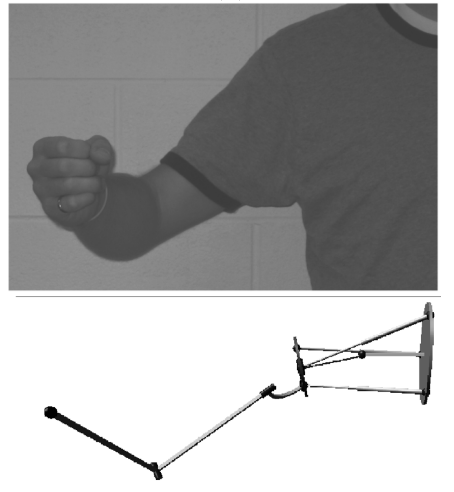

(d)

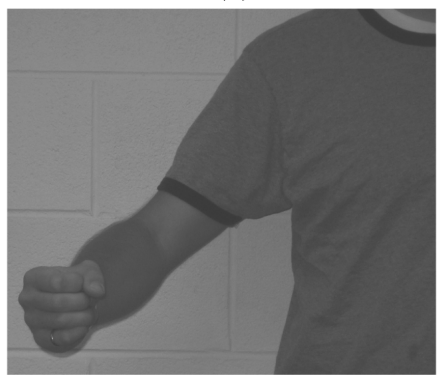

(e)
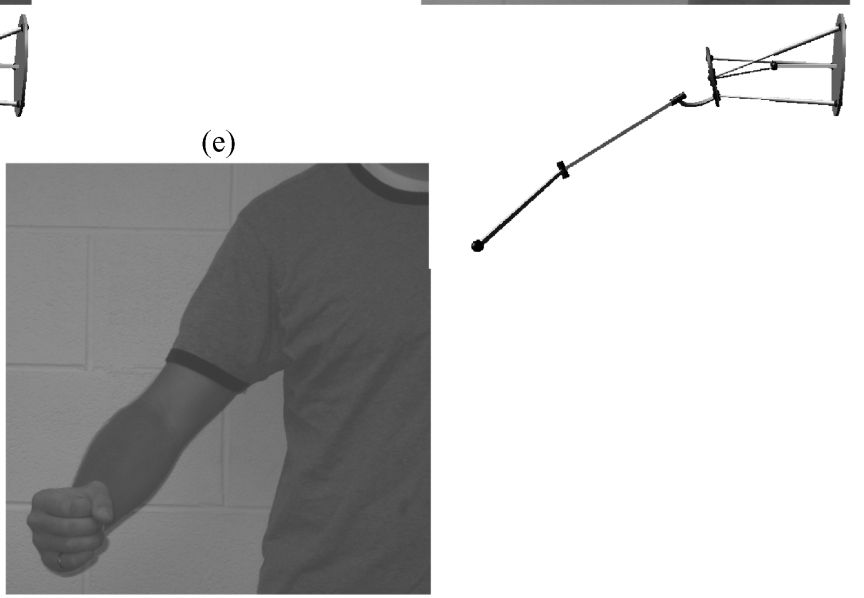

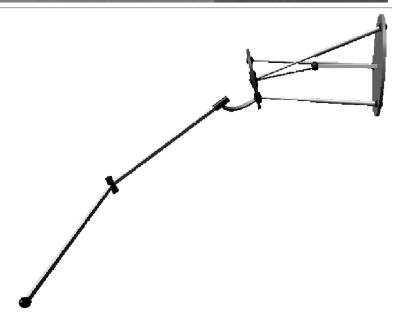

Figure 6. Vertical straight line trajectory, human motion versus humanoid motion.

easily observed by examining the best and worst data set comparisons used in the above study.

When the mechanism does not accurately match the position of the shoulder centre for a desired wrist position (the worst case scenario), there exists a difference between the reaching directions of $26.14^{\circ}$ with a difference between elbow axis directions of $48.54^{\circ}$. When the mechanism is able to reproduce a similar shoulder centre position for a desired wrist position (the best case scenario), there exists a difference between reaching directions of $0.58^{\circ}$ with a difference between elbow axis directions of $3.39^{\circ}$. 
(a)

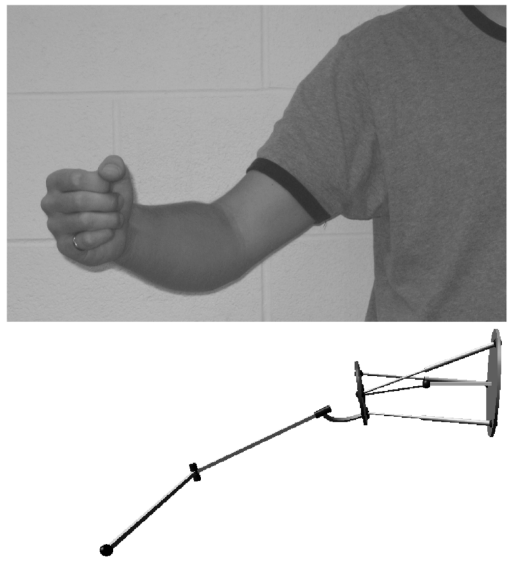

(c)

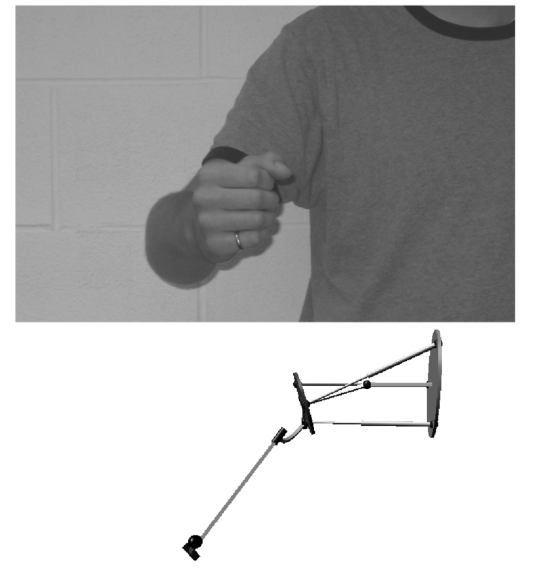

(b)

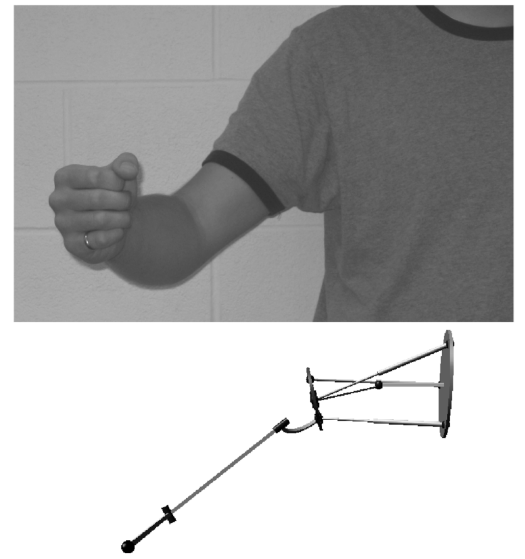

(d)
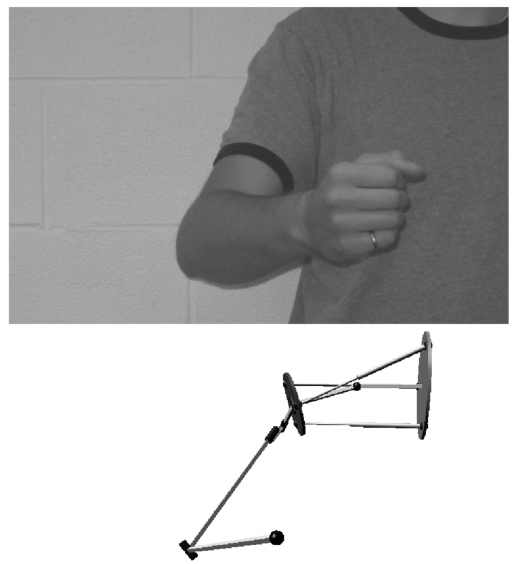

(e)

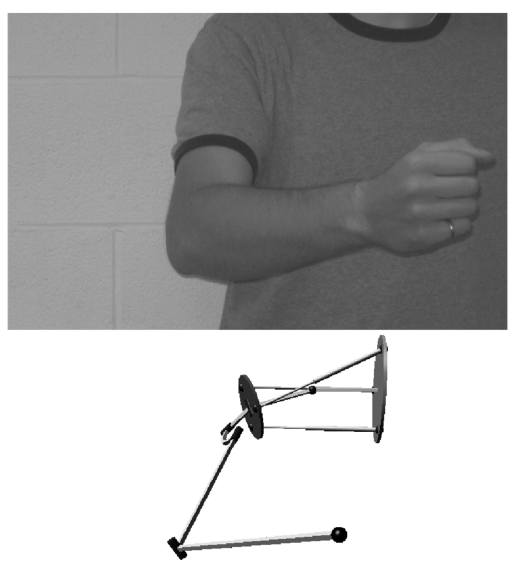

Figure 7. Straight line trajectory in the transverse plane, human motion versus humanoid motion.

This last result, when factoring in the errors that exist in the data collection and processing, shows that when the humanoid shoulder-elbow complex mechanism has a similar shoulder girdle configuration to that of a human, the difference in the respective arm configurations results in the elbow axis directions being off by only $1-2^{\circ}$.

\subsection{Qualitative comparison of humanoid mechanism motion to human motion}

In order to visually analyse the humanoid shoulder-elbow complex mechanism, a detailed three-dimensional animation was created using OpenGL (Open Graphics Library developed by Silicon Graphics Inc.). A static pose of the 
mechanism is displayed in Figure 5 as an example of the mechanism animation. Using this animation, it is possible to study arm configurations throughout different trajectories. The mechanism has been observed producing smooth, continuous motions that mimic motions of a human arm.

The animation of the humanoid shoulder-elbow complex mechanism was compared to voluntary humanreaching motion. A right-handed male subject of age 24 performed reaching trajectories in the anterior region of the body. The values for the constant $\mathrm{DH}$ parameters were roughly measured as

$$
\begin{gathered}
a_{0}=-115 \mathrm{~mm}, a_{2}=115 \mathrm{~mm}, d_{5}=320 \mathrm{~mm}, \\
\alpha_{5}=-102^{\circ}, a_{6}=300 \mathrm{~mm} .
\end{gathered}
$$

One of the sets of trajectory snap-shots is presented in Figure $6(\mathrm{a}-\mathrm{e})$. Here, both the human and the humanoid are moving their wrist on a vertical straight line. Another set of trajectory snap-shots is presented in Figure 7 (a-e). Here, both the human and the humanoid are moving their wrist on a horizontal straight line.

Overall, the humanoid shoulder-elbow complex mechanism was able to both quantitatively and qualitatively compare to actual human arm motion in an acceptable manner.

\section{Conclusion}

This paper presented an algorithm for the kinematic control of a humanoid shoulder-elbow complex mechanism that reproduces the range of motion of the human shoulder-elbow complex. The mechanism is an extension of the shoulder complex found in Lenarcic and Stanisic (2003) into a whole $\mathrm{arm}$. The kinematic solution replicates the motion of the human shoulder complex and closely approximates the coupling between the reaching direction and elbow axis direction for voluntary reaching motions. The algorithm used the method of successive iterations to solve the kinematically complex and coupled system. Replication of human shoulder-elbow complex motion was verified quantitatively and qualitatively.

\section{References}

Ambrose RO, Aldridge H, Askew RS, Burridge RR, Bluethmann W, Diftler M, Lovchik C, Magruder D, Rehnmark F, July/August, 2000. Robonaut: NASA's space humanoid IEEE J. Intell. Syst. 57-63.

Asfour T, Berns K, Dillmann R, 2000. The humanoid robot ARMAR: design and control. Proceeding. of the International Conference on Humanoid Robots, Boston. Vol. 1, pp. 1-6.

Craig JJ, 1989. Introduction to robotics: mechanics and control, 2nd Ed. New York: Addison-Wesley Publishing Company.

Dobrowolski JA, 1965. Completely automatic synthesis of optical thin film systems. Appl. Optics 4(8), August: 937-946.

Hashimoto S, Narita S, Kasahara H, Shirai K, Kobayashi T, Takanishi A, Sugano S, Yamaguchi J, Sawada H, Takanobu H, et al.
2002. Humanoid robots in waseda university - Hadaly-2 and WABIAN Autonom. Rob. 12: 25-38.

Ishida T, October/November, 2004. Development of a small biped entertainment robot QRIO. Proceeding of the International Symposium on Micro-Nanomechatronics and Human Science. pp. 23-28.

Kaneko K, Kanehiro F, Kajita S, Hirukawa H, Kawasaki T, Hirata M, Akachi K, Isozumi T, April, 2004. Humanoid Robot HRP-2. Proceedings of the IEEE International Conference on Robotics \& Automation, New Orleans, pp. 1083-1090.

Kaneko K, Kanehiro F, Kajita S, Yokoyama K, Akachi K, Kawasaki T, Ota S, Isozumi T, 2002. Design of prototype humanoid robotics platform for HRP. Proceedings of the IEEE/RSJ International Conference on Intelligent Robots and Systems, Switzerland. Vol. 2, pp. 2431-2436.

Klopcar N, Lenarcic J, 2001. Biomechanical considerations on the design of a humanoid shoulder girdle. Proceedings of the IEEE/ASME International Conference on Advanced Intelligent Mechatronics, Como. Vol. 1, pp. 255-259.

Lenarcic J, Stanisic M, 2003. A humanoid shoulder complex and the humeral pointing kinematics. IEEE Trans. Rob. Automat., 19(3): pp. 499-506.

Lenarcic J, Stanisic MM, Parenti-Castelli V, April, 2000. Kinematic design of a humanoid robotic shoulder complex. Proceedings of the IEEE International Conference on Robotics \& Automation, San Francisco, CA. pp. 27-32.

Miwa H, Itoh K, Ito D, Takanobu H, Takanashi A, April, 2004. Design and control of 9-DOFs emotion expression humanoid arm. Proceedings of the IEEE International Conference on Robotics \& Automation, New Orleans, LA., pp. 128-133.

Movellan JR, Tanaka F, Fortenberry B, Aisaka K, 2005. The RUBI/QRIO project: origins, principles, and first steps. Proceedings of 4th IEEE International Conference on Development and Learning, pp. 80-86.

Nakamura Y, Okada M, Shinohara T, Gotoh T, Ban S, 2002. Mechanical challenges for further humanoid robot evolution. Proceedings of the Third IARP International Workshop on Humanoid and Human Friendly Robotics.

Nickels KM, Baker K, August, 2003. Hand-eye calibration for robonaut. Final Report - NASA Faculty Fellowship Program. Contract No. NAG9-1526, pp. 13-1-13-15.

Nishiwaki K, Sugihara T, Kagami S, Kanehiro F, Inaba M, Inoue H, 2000. Design and development of research platform for perception-action integration in humanoid robot: H6. Proceedings of the IEEE/RSJ International Conference on Intelligent Robots and Systems, pp. 1559-1564.

Oatis CA, 2004. Kinesiology: The mechanics and pathomechanics of human movement. Philadelphia, PA: lippincott, Williams and Wilkins.

Okada M, Nakamura Y, Ban S, May, 2001. Design of programmable passive compliance shoulder mechanism, Proceedings of the IEEE International Conference on Robotics \& Automation, Seoul, pp. 348-353.

Okada M, Nakamura Y, Hoshino S, 1999. Development of the cybernetic shoulder - a three DOF mechanism that imitates biological shoulder-motion. Proceedings of IEEE/RSJ International Conference on Intelligent Robots and Systems. Vol. 2, pp. 543-548.

Okada M, Shinohara T, Gotoh T, Ban S, Nakamura Y, March, 2003. Humanoid robot mechanisms for responsive mobility. Proceedings of the 2nd International symposium of Adaptive Motion of Animals and Machines, Kyoto.

Rosheim ME, June, 1997. In the footsteps of leonardo. IEEE Rob. Automat. Mag., pp. 12-14.

Sawada T, Takagi T, Fujita M, September/October, 2004. Behavior selection and motion modulation in emotionally grounded 
architecture for QRIO SDR-4XII. Proceedings of IEEE/RSJ International Conference on Intelligent Robots and Systems, Sendai, pp. 2514-2519.

Sodeyama Y, Mizuuchi I, Yoshikai T, Nakanishi Y, Inaba M, August, 2005. A shoulder structure of muscle-driven humanoid with shoulder-blades. IEEE/RSJ International Conference on Intelligent Robots and Systems, pp. 4028-4033.

Stanisic MM, Goehler CM, Tomsic M, 2007, A quantified description of the coupling between the reaching direction and elbow axis direction. Appl. Bion. Biomech. 4(2): 47-55.

Stanisic MM, Wiitala JM, Feix JT, 2001. A dexterous humanoid shoulder mechanism. J. Rob. Syst. 18(12): 737745.

Tanaka F, Fortenberry B, Aisaka K, Movellan JR, 2005. Plans for developing real-time dance interaction between QRIO and toddlers in a classroom environment. Proceedings of the 4th IEEE International Conference on Development and Learning, pp. 142-147.

Tanaka F, Suzuki H, September, 2004. Dance interaction with QRIO: A case study for non-boring interaction by using an entertainment ensemble model. Proceedings of the IEEE International Workshop on Robot and Human Interactive Communication, Kurashiki, Okayama. pp. 419-424.

Zatsiorsky VM, 1998. Kinematics of human motion Champaign, IL:, Human Kinetics.

Zollo L, Siciliano B, Laschi C, Teti G, Dario P, 2002. Compliant control for a cable-actuated anthropomorphic robot arm: an experimental validation of different solutions. Proceedings of the IEEE International Conference on Robotics \& Automation, Washington, D.C, Vol. 2, pp. 1836-1841. 

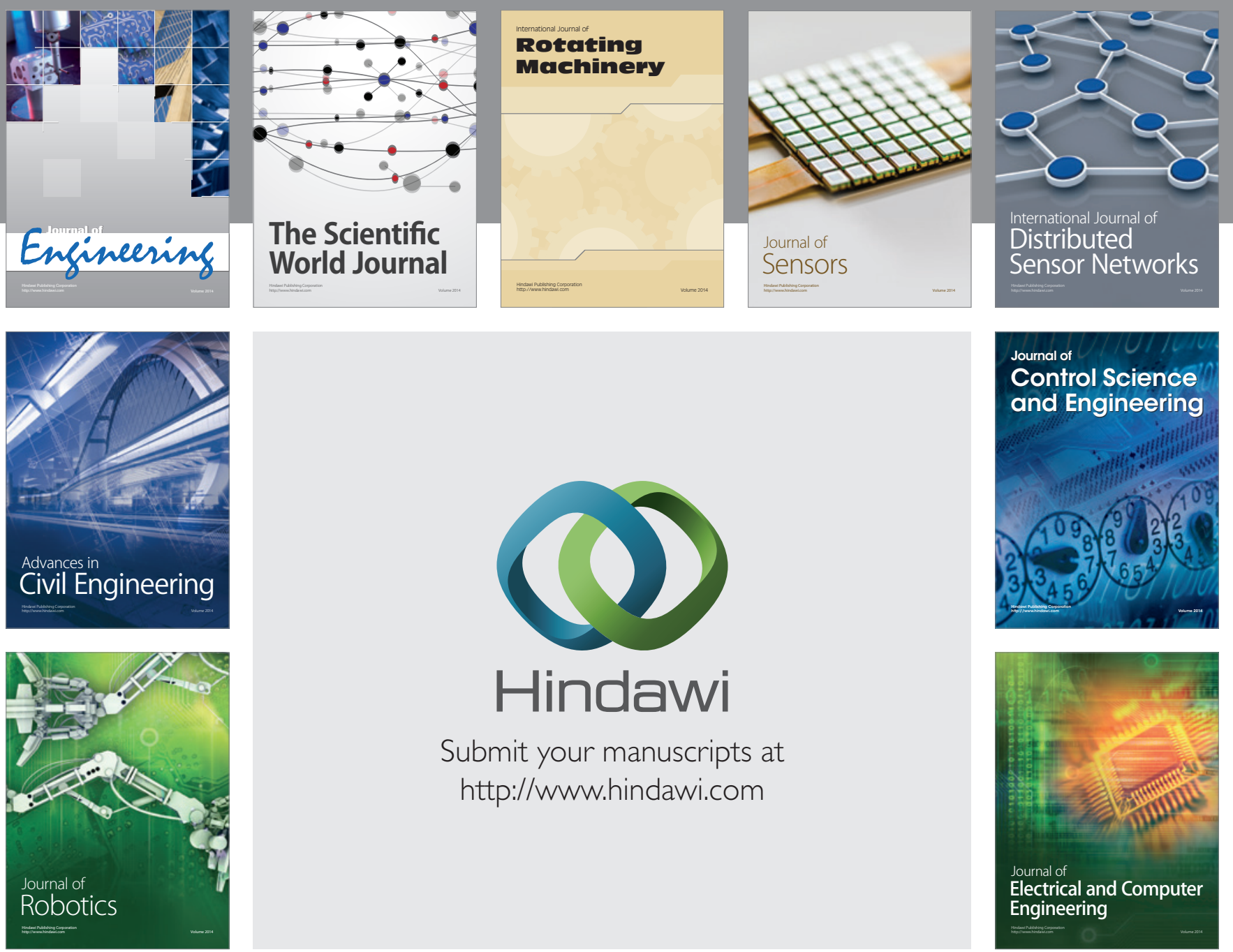

Submit your manuscripts at

http://www.hindawi.com
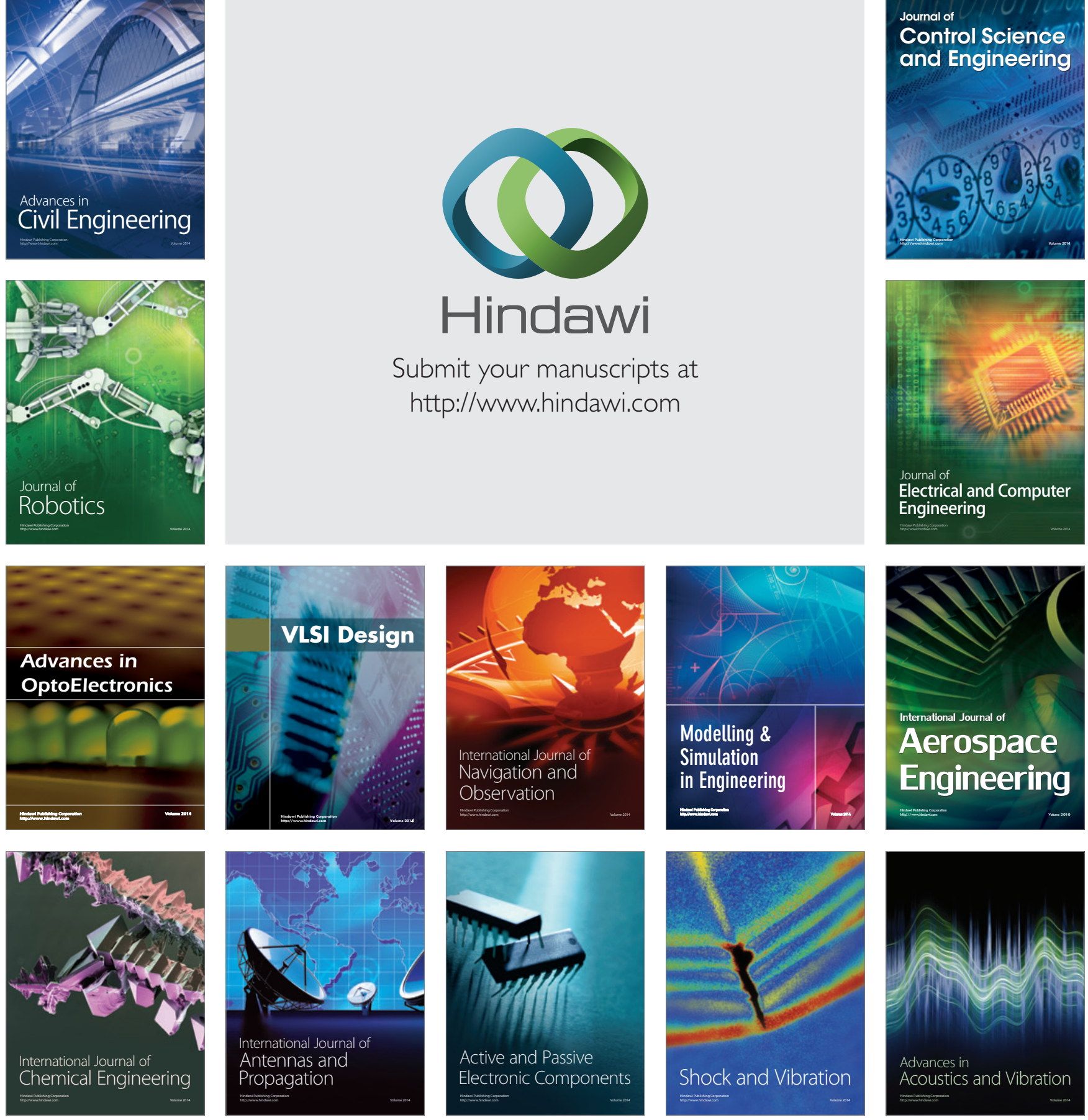